\title{
A Quadruped Robot for RoboCup Legged Robot Challenge in Paris '98
}

\author{
Masahiro Fujita ${ }^{1}$, Stephane Zrehen ${ }^{1}$ and Hiroaki Kitano ${ }^{2}$ \\ 1 D21 Laboratory, Sony Corporation, \\ 6-7-35, Kitashinagawa, \\ Shinagawa-ku, Tokyo, 141 JAPAN \\ 2 Sony Computer Science Laboratory Inc. \\ Takanawa Muse Building, 3-14-13 Higashi-gotanda, \\ Shinagawa-ku, Tokyo, 141 JAPAN
}

\begin{abstract}
One of the ultimate dream in robotics is to create life-like robotics systems, such as humanoid robot and animal-like legged robot. We choose to build pet-type legged robot because we believe that dog-like and cat-like legged robot has major potential for future entertainment robotics markets for personal robots. However, numbers of challenges exists before any of such robot to be fielded in the real world. Robots have to be reasonably intelligent, maintains certain level of agility, and be able to engaged in some collaborative behaviors. RoboCup is an ideal challenge to foster robotics technologies for small personal and mobile robotics system. This paper, we present Sony's legged robots system which enter RoboCup-98 Paris as a special exhibition games.
\end{abstract}

\section{Introduction}

Robot systems with life-like appearance and behaviors are one of the ultimate dream in robotics and AI [Inaba, 1993, Maes, 1995]. Honda's Humanoid Robot announced in early 1997 clearly demonstrated that it is technically feasible and we have all recognized that social and psychological impacts of such technologies are far reaching. A robot system described in this paper presents our effort to build reconfigurable robot system that has high degree of design flexibility. While many robotics system uses wheel-based driving mechanisms, we choose to develop legged robot which can be converted into wheel-based robots easily by using reconfigurable physical components. Among various types of robot configurations attainable using our architecture, our immediate focus is a legged robot configuration with four legs and a head each of which has three degree of freedom. Given that our goal is to establish Robot Entertainment industry, this configuration is attractive because it resembles dogs or cats, so that people may view it as robot pets. It also entails numbers of technical issues in controlling its motion, while avoiding difficulties of biped robot systems.

We are interested in participating in RoboCup, Robot World Cup Initiative [Kitano, et al., 1997], using our legged robots, as a part of its exhibition program. RoboCup is an ideal forum for promoting intelligent robotics technologies because it offer challenging and exciting task of playing soccer/football games by 
multiple robots. In order for a team of robots to play soccer game, robots should be able to identify environment in real-time under less controlled world than the laboratory set up, move quickly, and has to have certain level of intelligence. For our legged robots, RoboCup would be even more challenging than wheel-based robots because participation to RoboCup requires sophisticated control system for 15 degree-of-freedom for multiple robots. In addition, body posture and head position moves constantly due to legged locomotion. This imposes serious problem for a vision system which acts as a central sensor system to identify ball position, opponent position, and position of goals.

All these challenges actually represents basic requirements for developing robot for personal use, especially for entertainment robots. For robot entertainment, each robot has to be fully autonomous, and it should be exhibit reasonable intelligence and maneuvering capability. For example, a robot dog should be able to identify the owner and chase a ball, and play with it. If unwanted person or objects show up in front of it, a robot dog should react quickly to escape from it. Often, few robot dogs may chase an emery as a team. Clearly, these basic elements can be found in RoboCup games. Of course, legged robot to play soccer game constitutes only a part of robot entertainment market. However, it can be a clear and present new market and it is a good starting point.

The legged robot described in this paper is based on OPEN-R standard ${ }^{3}$, which is designed as a standard architecture and interfaces for Robot Entertainment [Fujita and Kageyama, 1997a]. OPEN-R aims at defining a standard whereby various physical and software components which meet the OPEN-R standard can be assembled with no further modifications, so that various robots can be created easily. We developed the legged robot platform based on the OPEN-R, and collaborating with three universities, we had an RoboCup Legged Robot Exhibition at Paris.

In this paper, first, we will describe the OPEN-R briefly, followed by description of the legged robot platform for RoboCup-98 Paris. Then, the field setup and the rule will be explained. Since the legged robot platform equipped with a color processing engine, the important items in the field were painted by different colors, which we selected carefully based on experiments. We will describe how we selected the colors, and how the robot can self-localize in the field. Finally, we will report the result of Legged Robot Exhibition at RoboCup-98 Paris with a brief introduction of features of each team.

\section{OPEN-R Standard}

\subsection{Overview}

OPEN-R is a standard for robot entertainment which enables reconfiguration of robot structure, as well as easy replacement of software and hardware components.

${ }^{3}$ OPEN-R is a trade mark of Sony Corporation. 
One of the purposes of proposing OPEN-R is that we wish to further promote research in intelligent robotics by providing off-the-shelf components and basic robot systems. These robots should be highly reliable and flexible, so that researchers can concentrate on the aspect of intelligence, rather than spending a substantial proportion of their time on hardware troubleshooting.
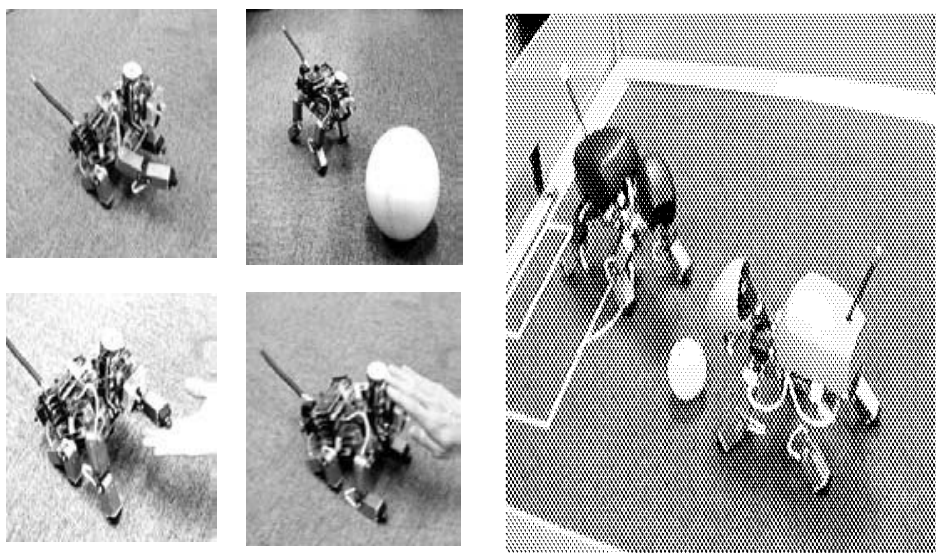

(a)

(b)

Fig. 1. MUTANT: (a) Fully autonomous pet-type robot and (b) remote-operated soccer game robots

For the feasibility study of OPEN-R, we developed an autonomous quadruped pet-type robot named MUTANT[Fujita and Kageyama, 1997a] (Fig.1 (a)). We also implemented and tested a remote-operated robot system for soccer game (Fig.1 (b)). Using these robots, software and application feasibility studies were carried out.

The legged robot for RoboCup described in is paper is a direct descendent of MUTANT and remote control soccer robots. The legged robot for RoboCup is a fully autonomous soccer robot.

\subsection{Major Features}

One of the salient feature of the OPEN-R standard is that is attains several critical dimensions of scalability. These are (1) size scalability, (2) category scalability, (3) time scalability, and (4) user expertise scalability. 
Size Scalability (Extensibility): OPEN-R is extensible for various system configuration. For examples, in a minimum configuration, a robot may be composed of only few components, and perform a set of behaviors as a complete agent. This robot can be scaled up by adding additional physical components. It is possible to scale up such a system by having such robots as sub-systems of large robot systems.

Category Scalability (Flexibility): Category scalability ensures that various kinds of robots can be designed based on the OPEN-R standard. For example, two very different styles of robots, such as a wheel-based robot and a quadruped-legged robot, should be able to described by the OPEN-R standard. These robots may have various sensors, such as cameras, infra-red sensors, and touch sensors and motor controllers.

Time Scalability (Upgradability): OPEN-R can evolve together with the progress of hardware and software technologies. Thus, it must maintain a modular organization so that each component can be replaced with up-to-date modules.

User Expertise Scalability (Friendly Development Environment): OPEN-R provides a development environments, both for professional developers and for endusers who do not have technical knowledge. End users may develop or compose their own programs using the development environment. Thus, it is scalable in terms of the level of expertise that designers of the robot have.

\subsection{OPEN-R Strategy}

Our strategy to meet these requirements consists of the following:

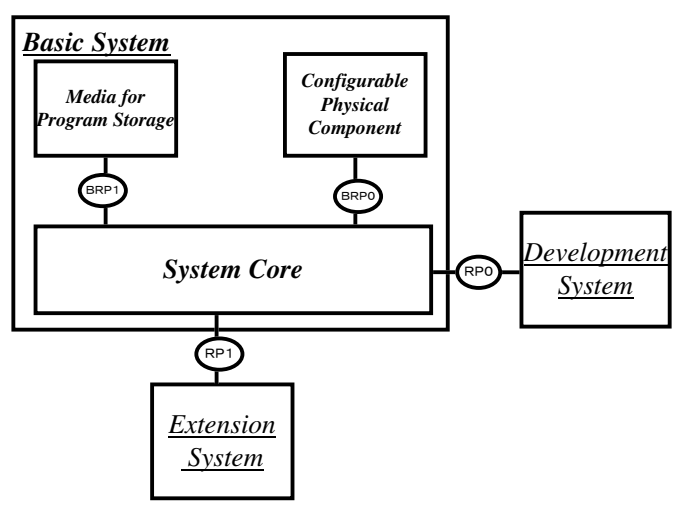

Fig. 2. Generic System Functional Reference Model and Examples of Derived System Architectures 
Generic Reference Model: To meet the requirements of Extensibility and Friendly Development Environment, we define a generic system functional reference model (GSFRM) composed of Basic System, Extension System and Development System. By defining GSFRM, we are able to construct various kinds of robot systems with extensibility and development environments, as shown in Fig.2.

Configurable Physical Component: To meet the requirements of Flexibility and Extensibility, we devise a new idea of Configurable Physical Component $(\mathrm{CPC})$. The physical connection between the robot components is done by a serial bus. In addition every CPC has non-volatile memory with (1) functional properties, such as an actuator and a two dimensional image sensor, and (2) physical properties, which help solve the dynamics of the robot consisting of these CPCs. Fig. 3 illustrate this concept.

Object-Oriented Programming: To meet the requirements of Up-gradability and Flexibility, we employ an object-oriented OS, Aperios [Yokote, 1992], which supports the Object-Oriented Programming (OOP) paradigm from the system level with several types of message passing among objects. In addition, Aperios is capable of customizing APIs by system designers.

Layering: To meet the requirements of Up-gradability and Friendly Development Environment, we utilize the layering technique which is often used for multi-vendor open architecture. OPEN-R divides each functional element into three layers, Hardware Abstraction Layer (HAL), System Service Layer (SSL), and Application Layer (APL).

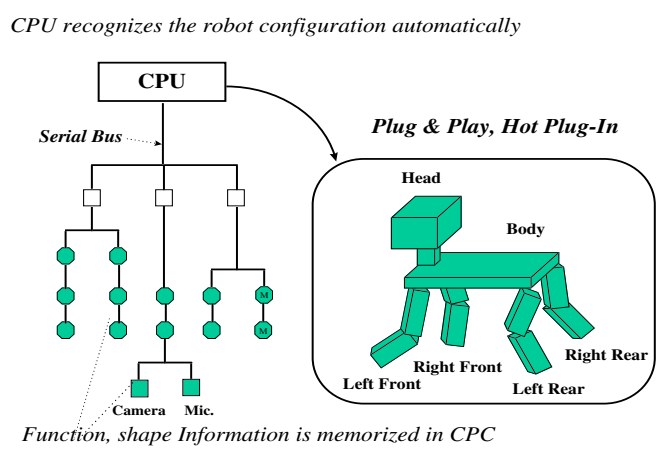

Fig. 3. Configurable Physical Component

In order to achieve the software component concept, an Agent Architecture for entertainment application was studied. The details is described in [Fujita and Kageyama, 1997a]. 


\section{Legged Robot Challenge}

A team of legged robot soccer players is a major challenge. It must address various issues involving control of robot with multiple degree of freedoms and stabilization and compensation of images obtained from continuously dislocating vision system due to legged locomotion, and other issues, in addition to all difficult problems offered in RoboCup Challenge for wheel-based robots.

\subsection{Legged Robot Platform for RoboCup}

Quadruped Legged Robot: For RoboCup-98 Paris, we will deploy legged robot with four legs and one head, each of which has three degree of freedom, and rich sensory channels, for example, a head has a color CCD camera, stereo microphone, touch sensors, and a loud speaker.

Most of the intelligent autonomous robots are implemented in wheel-based mechanical configuration. A wheel-based robot has advantage in their simplicity of motion control, so that researchers can concentrate on vision, planning, and other high-level issues. However, since our goal is robot entertainment, different emphasis shall be made. We believe that the capability of representation and communication using gesture and motion is very important in entertainment applications. Therefore, we choose a mechanical configuration of our robot as a quadruped-legged type, as shown in Fig.4.

The merits of the quadruped-legged configuration are, (1) walking control of a quadruped-legged is easier than that of a biped robot, and (2) when in a posture of sitting, two hands are free to move, therefore, they can be used to present emotions or to communicate with a human by the motions of the hands. Since each leg or hand has to be used for various purposes besides walking, we assign three degree of freedom (DoF) for each leg/hand. In addition, we add a tail and three DoF for neck/head so that the robot has enough representation and communication capabilities using motions.

During the RoboCup games, legs are not necessary used for expressing emotions. However, they can be used for sophisticated control of balls, such as passing ball to the side or back, or engaged in deceptive motions.

Disadvantages of using legged robot is their moving speed is not as fast as wheel-based robots. In future, speed issue may be resolved when galloping was made possible. For now, legged robot will be played within dedicated league. Although serious hardware limitation exists, teams with efficient leg motion coordination will have major advantages in the game.

Standalone System: In general, it is difficult for a standalone robot system to perform these tasks in real time in a real world environment because of its limited computational power. The remote operated robot system depicted in Fig.2 can solve the computational power problem; however, in general, much computational power is necessary for image processing tasks. This implies that it is necessary for each robot to be equipped with a video transmitter. This is sometimes difficult for regulation reason. 


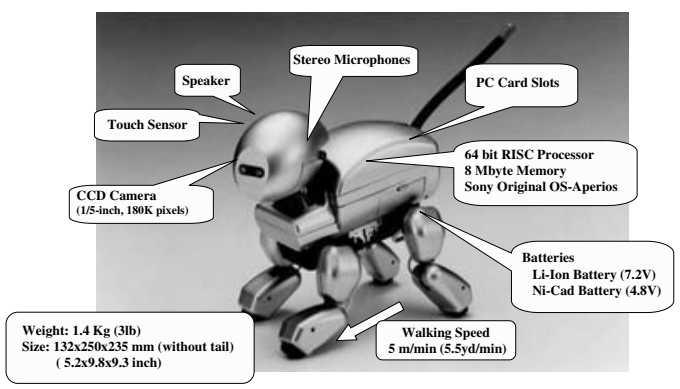

Fig. 4. Mechanical Configuration

Another solution to this problem is to set up a camera overlooking the entire field, and to distribute the image signal to all host computers. In this set up, each robot can use the huge computer power of the host computer, or a special engine such as image processing hardware. However, the image information taken by the overlooking camera is not the image taken from each robot's viewpoint.

We consider that technologies to process the image from each robot viewpoint without any global information will become very important in Robot Entertainment in future. Therefore, we decide to build RoboCup System with standalone robots under local communication constraint.

There are two hardware issues that need to be resolved to enable full onboard vision system for small size robots: (1) camera size, and (2) processor power. We solved these problem by actually manufacturing a dedicated camera and a processor chip.

Micro-Camera-Unit: In order to make a robot small in size and weight and to reduce cost, we developed a Micro-Camera-Unit (MCU) using multi-chipmodule technology ([Ueda, 1996]), as shown in Fig. 5. This MCU includes a lens in the same package to achieve a single thin camera module. The size of the MCU is $23 \times 16 \times 4 \mathrm{~mm}$, with pixels $362 \times 492$.

OPEN-R Chip: To make the robot small in size and in power consumption, we employ MIPS architecture's R4000-series CPU with more than 100 MIPS performance. We have also developed the dedicated ASICs including the peripheral controllers of the CPU, as described before. Fig. 6 shows the entire electrical block diagram, where we employ the Unified Memory Architecture with synchronous DRAMs (SDRAM) as a main memory. The features of the ASIC are as follows:

OPEN-R BUS controller: The OPEN-R bus controller controls all CPC 


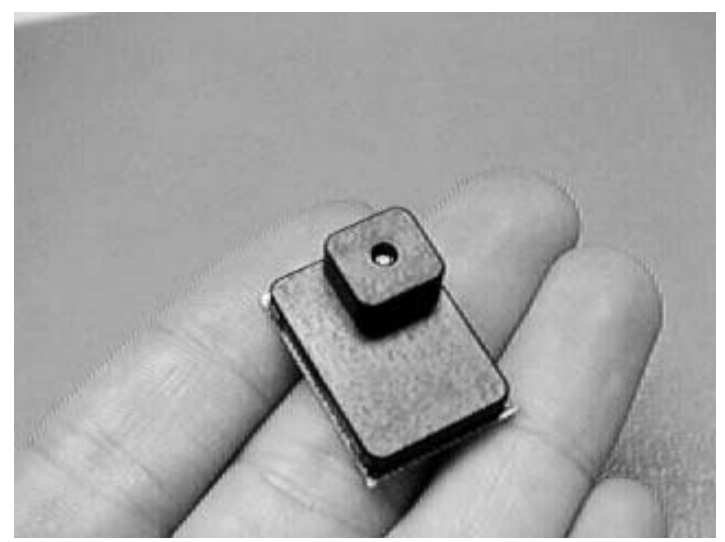

Fig. 5. Micro Camera Module

devices which are connected in tree structure fashion (Fig.3).

Image Signal Processing Engine: The Camera data is transferred to the SDRAM through the multi-resolution filter bank (FBK). This FBK consists of three layer resolution filters. The resolutions of the filters are $360 \times 240,180 \times 120$ and $90 \times 60$. The filtered image data through the FBK can be applied by the color detection engine (CDT) so that eight color can be detected in real-time.

DSP: For sound processing, the integer DSP with about $50 \mathrm{MHz}$ clock is integrated in the ASIC so that the FFT or filter bank processing for sound data can be done in real-time.

Although dedicated camera module and OPEN-R Chip enables on-board vision processing, it still requires major efforts to recognize natural images of balls and field. However, color marking regulation of RoboCup enable us to identify goals, a ball, and other robots using Color Detection Engine.

\section{$3.2 \quad$ Soccer Field}

The size of the soccer field for the quadruped robot competition is shown in Fig.7. This size is not the same as the official filed size of RoboCup Small Size League or Middle Size League. We considered three players by three players competition, and this size is considered based on the size of our robot.

Each wall is slanted by 45 degree with $10 \mathrm{~cm}$ height. This slant keeps a distance between a ball and the wall so that a robot player can easily kick the ball. In addition, the corner has a triangle slant wall. This also avoids the ball stacking in the corner.

As we mentioned above, the items in the field are painted by carefully selected colors. The color assignment is shown in Fig.8. 


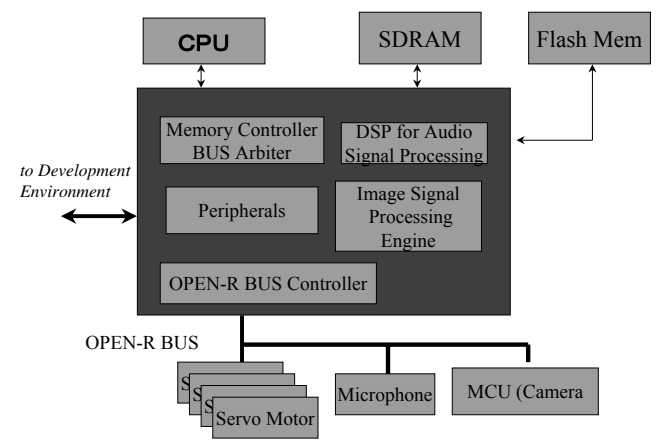

Fig. 6. Electrical Block Diagram including the ASIC

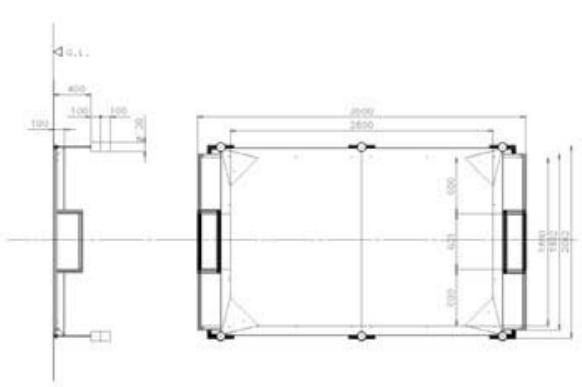

Fig. 7. A dimension of the Soccer Field

The six poles with two colors are considered to be used for landmarks, which can be used for self-localization in team collaboration strategy described in the following section.

The color samples used in the field is shown in Fig.9. The image was taken by a robot camera with a lighting condition of $580(l x)$ measured by an illuminometer, and a color temperature of $4500(K)$ measured by a CIE 1931 chromaticity meter. 


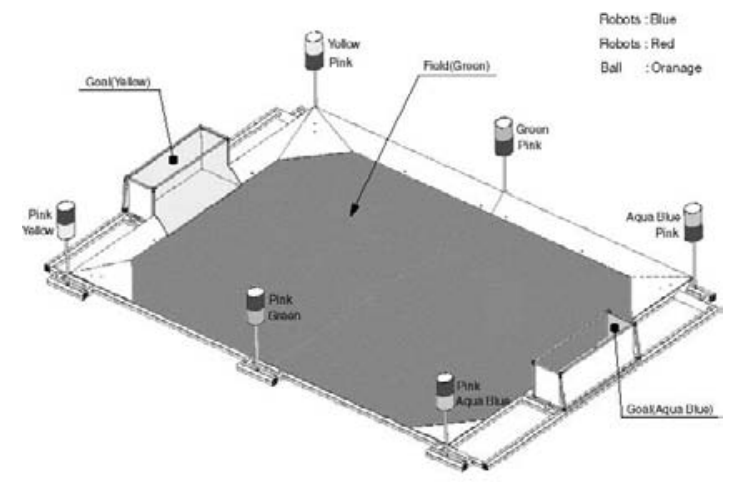

Fig. 8. Color assignment in the field

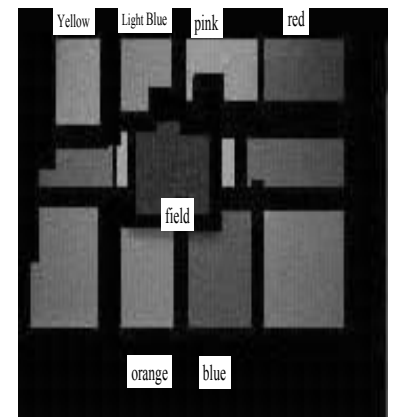

(a)

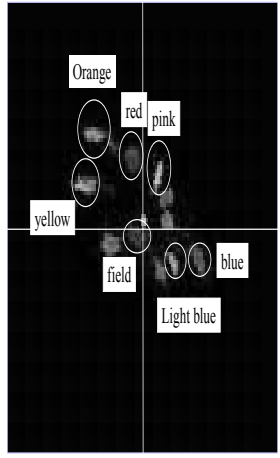

(b)

Fig. 9. (a) Color samples used in the field. and (b) its distribution in the UV plane 


\section{Vision Subsystem}

While there are numbers of software issues exist, this paper briefly describe vision subsystem to detect and track objects, and to localize robot's own position.

\subsection{Object Tracking and Distance Estimation}

There are few objects which need to be identified in RoboCup games - goals, a ball, and other robots. Basically, each object can be identified with color. A ball is painted in a single color, and each goal is painted in a distinguishable color. Robots are basically painted in black or a dark color. We use color (red and blue) painted costume so that a robot can recognize opponent players. Obviously, the most important object is a ball. This can be done in rather simple manner, that to find a define color for the ball, e.g. red. To keep the ball in the center of the image, the robot head direction is feedback controlled, and the walking direction is also controlled using the horizontal head direction. The advantage of having a neck is that the robot can continue to keep the ball within visual field even when the robot do not walk toward the ball. These head position control is carried out by neck-subsystem using behavior-based approach [Brooks, 1986]. Our distributed layering of agent architecture, similar to [Firby, 1994] enables such an implementation.

Both the horizontal and vertical neck angle can be utilized to estimate the distance between the ball and the robot body (Fig.10). This information is also used as a queue to execute a kick motion.

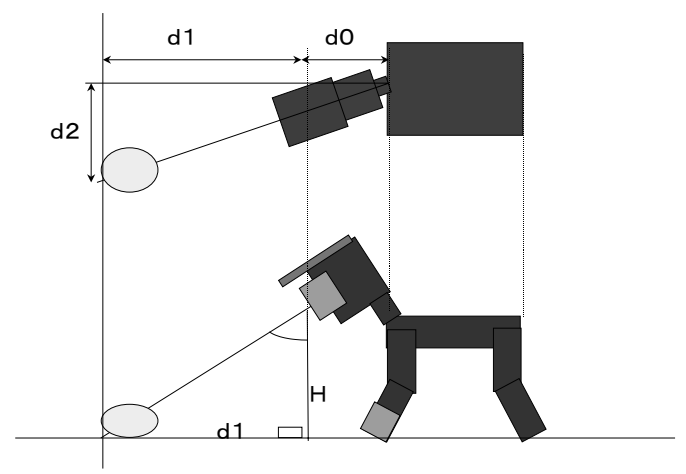

Fig. 10. Ball Positioning

Assume that the robot knows $H$, the height of its camera view point from the ground, following procedure identifies distance of the robot from the ball. 
1. Find the ball.

2. Measure the neck's vertical angle facing the ball $(\alpha)$, and horizontal angle $(\theta)$.

3. $\operatorname{Han}^{-1}(\alpha)$ is the distance from the ball along the axis of body's moving direction (d1), and $\tan ^{-1}(\theta)$ is the displacement of the ball from the axis of body direction (d2).

\subsection{Self-localization through vision}

Visual landmarks on the walls of the field can be used for self-localization purposes. Indeed, any x-y position can be computed by triangulation of three landmarks azimuths, assuming that the absolute position of landmarks is known. In order to limit the computational demands on the CPU for visual processing and to make exhaustive use of the color processing hardware provided with the camera module, we will paint visual landmarks regularly along the walls of the field. These landmarks are represented on figure 11 . They are about $10 \mathrm{~cm}$ high and $3 \mathrm{~cm}$ wide. They are composed of three color strips, and are limited on their top and their bottom by bright yellow bands. The information carrying colors are chosen among four: blue, pink, yellow and indigo. The bottom color indicates the wall on which the landmark is located.

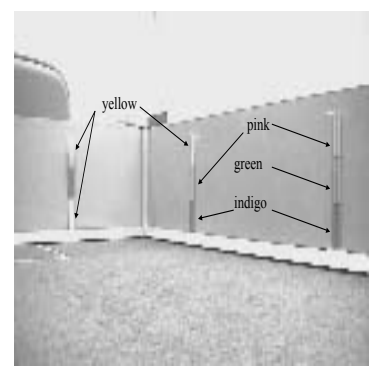

(a)

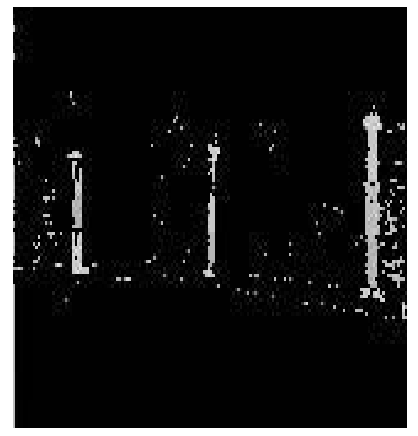

(b)

Fig. 11. (a) an image of two color landmarks pasted on the field walls, and (b) the result of color detection 
The special color processing hardware allows the detection of 8 template colors and provides output in the form of 8 bit-planes, for every field, that is, every $50 \mathrm{~ms}$. Color detection proceeds as follows: The input image is represented in the YUV space, using 256 values for $\mathrm{U}$ and $\mathrm{V}$ and 32 values for $\mathrm{Y}$. A lookup table is established for every template color in the form of a cube in the YUV space, the dimensions of which representing a matching threshold on color comparison. In our preliminary studies, using landmarks printed on a simple color printer and then pasted on the walls with yellow adhesive tapes, it appears that it is best to use two templates for each color, one for the lighter regions and one for the darker ones. This is notably due to the presence of shadows whose color is hard to model. An example of color detection is presented on Figure 11 (b). The image is noisy, due to the existence of reflections on the plastic walls and to the absence of any noise-removing routine. However, this is not a major problem since in the localization subsystem we are only trying to identify and localize the landmarks in the visual field, and not to exhaustively segment the image. Landmark detection and identification is performed by detecting three small color squares in a vertical arrangement. On a sequence of 10 seconds where a -90 to +90 degrees motion was performed, all the landmarks but one were correctly identified. In such cases, the information extracted from the sequence can help overcome the wrong detection.

Since the micro-camera module has a limited visual angle, a head rotation movement might be necessary to identify and localize distant landmarks, for good resolution in the triangulation computation. This is time consuming and may not always be necessary. Perspective information can be used to obtain qualitative information about the robot's position in the field. For instance, on Figure 11 (a), the relative size of the two landmarks on the right tells that the right wall of the field is on the right side of the robot. On the other hand the similar size of the two landmarks on the left tells that the robot is facing a corner since these two landmarks are known to be on different walls. In addition, it is possible to establish a lookup table of the visual size of a landmark as a function of its distance to the robot.

The use of this system will thus make self-localization possible and easy without requiring any global positioning system, which would need to be outside the robot.

\section{$5 \quad$ Report of RoboCup 98 Paris}

In this section we present some of the work developed with the legged robot platform at Carnegie Mellon University (CMU), in the Laboratoire de Robotique de Paris (LRP), and at Osaka University.

\subsection{Participants}

CMU: CMTrio: CMU had decomposed their work along the following aspects: 
- Reliable detection of all of the relevant colors: orange (ball), light blue (goal and marker), yellow (goal and marker), pink (marker), light green (marker), dark blue (teammate/opponent), and drak red (opponent/teammate).

- Active ball chasing: the robot activity interleaves searching for the ball and localization on the field to evaluate both an appropriate path to the ball and final positioning next to the ball.

- Game-playing behaviors: robots play attacking and goal keeping positions.

They use supervised learning for color detection and Bayesian Estimation for Self-locarization. The main feature of CMTrio was stable visual perception. Since the basic behaviors of the player in the color painted field were based on the visual perception, the stability of the color detection is one of the most important points in the Legged Robot Exibition.

LRP: Les Titis Parisiens: The French team had focused on the three points:

- Vision Recognition System

- The design of walking patterns

- The strategy chosen to play soccer games

The most important feature of the French team was the development of walking pattern. Although Sony provided a basic walking generation software, only this team developed the walking pattern by themselves, which was very stable and fast.

Osaka University: Baby Tigers: The main feature of Osaka University team was the development of the metholodogy to acquire basic behaviors from the interactions between the legged robots through multi sensor-motor coordinations. The behaviors of Baby Tigers were not Top-down programmed, but teached by presenting many sets of visual patterns and behavior commands.

\subsection{Results}

The event schedule is shown in Fig. 12.

From July 4th to 5 th, we had non-official competitions, but only practical purpose games. On July 6th, it was not opened to public, but only to RoboCup participants. From July 7 th to 8 th, we had official competitions. The result of round-robin was shown in Fig.13.

\section{Conclusion}

Building a reliable and powerful robot platform meeting commercial-level product reliability is a challenging task. A small-size legged robot is even more challenging. We managed to produce such a robot platform by actually fabricating 
Time Schedule of "the Legged Robot Exhibition"
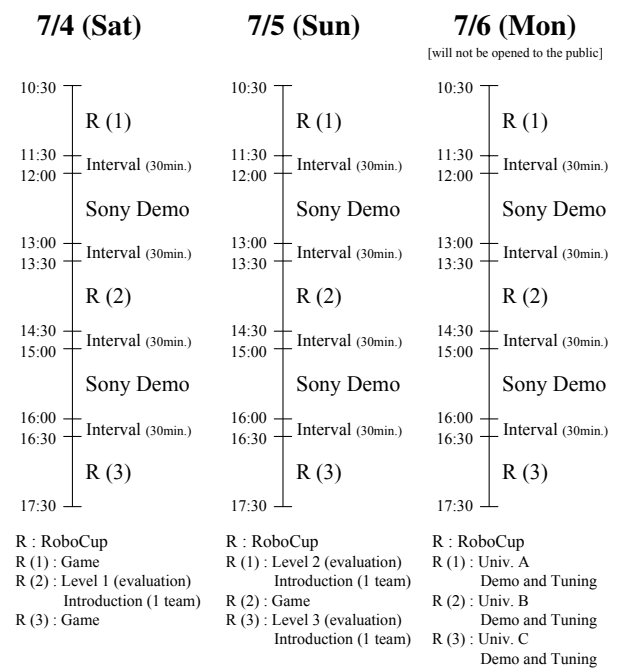

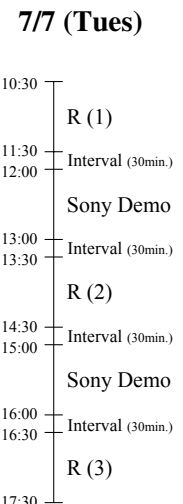

$\mathrm{R}$ : RoboCup

$R(1)$ : Game

$\mathrm{R}(2)$ : Level 1 (evaluation)

Introduction $(1$ team $)$

$R$ (3) : Game
$7 / 8$ (Wed)

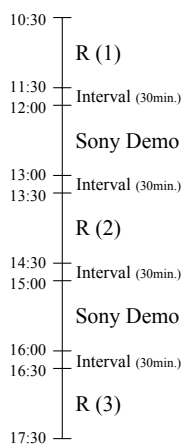

$\mathrm{R}$ : RoboCup

$\mathrm{R}$ (1) : Level 2 (evaluation) Introduction (1 team)

R (2) : Game
R (3) : Level 3 (evaluation) Introduction (1 team)

Fig. 12. Schedule of RoboCup-98 Paris: Legged Robot Exhibition

RoboCup Legged Robot Exhibition Match

\begin{tabular}{|c|c|c|c|c|}
\hline & $\begin{array}{c}\text { CM-Trio } \\
\text { CMU }\end{array}$ & $\begin{array}{c}\text { Les Titis } \\
\text { Parisiens } \\
\text { LRP }\end{array}$ & $\begin{array}{c}\text { Baby Tigers } \\
\text { OSAKA }\end{array}$ & $\begin{array}{c}\text { Total Game } \\
\text { Point }\end{array}$ \\
\hline $\begin{array}{c}\text { CM-Trio } \\
\text { CMU }\end{array}$ & $2-1$ & $1-1$ & 5 \\
\hline $\begin{array}{c}\text { Les Titis } \\
\text { Parisiens } \\
\text { LRP }\end{array}$ & $1-2$ & $1-0$ & 3 \\
\hline $\begin{array}{c}\text { Baby Tigers } \\
\text { OSAKA }\end{array}$ & $1-1$ & $0-1$ & 1 \\
\hline
\end{tabular}

Fig. 13. Result of Competitions 
dedicated components, such as a micro camera unit, a special chip, and mechanical/electrical joints. These specification of robot were now being compiled as OPEN-R standard. OPEN-R will be able to supply broad range of useful mechanical and electrical hardware and software components.

Nevertheless, software to control such a robot and to make them behave to carry out meaningful tasks is major research topic. In order to facilitate research in the control of legged robot with multiple degree of freedom and rich sensor systems, we choose to developed a physical robot platform for RoboCup based on OPEN-R, and to participate in RoboCup.

\section{Acknowledgements}

We thank to the RoboCup98 Legged Robot Challengers at CMU, LRP, and Osaka University. Especially we would like to thank to Dr. Veloso, Dr. Duhaut, and Dr. Asada. Without their efforts, we couldn't have exciting competition games during the short period of development. The authors also would like to thank to Ms. Aline Dahlke at Sony France and Ms. Asako Watanabe at D21 Laboratory, Sony Tokyo. Had it not been for their support and preparation, the event "RoboCup-98 Legged Robot Exhibition ! Ivould not have been a big success like that.

\section{References}

[Brooks, 1986] Brooks, R. A., 1986, A Robust Layered Control System for a Mobile Robot, IEEE Journal of Robotics and Automation, RA-2(1), March, pp.14-23.

[Firby, 1994] Firby, R. J., 1994, Task Networks for Controlling Continuous Processes, In Proceedings on the Second International Conference on AI Planing Systems, June.

[Fujita and Kageyama, 1997a] Fujita, M. and Kageyama, K., 1997, An Open Architecture for Robot Entertainment, In Proceedings of the First International Conference on Autonomous Agents, Marina del Ray, pp.234-239.

[Inaba, 1993] Inaba, M., 1993, "Remote-Brained Robotics: Interfacing AI with Real World Behaviors, In Proceedings of the 6th International Symposium on Robotics Research (ISRR6), pp.335-344.

[Kitano, et al., 1997] Kitano, H., et al, 1997, RoboCup: A Challenge Problem for AI, AI Magazine, Spring, pp.73-85.

[Maes, 1995] Maes, P., 1995, Artificial Life meets Entertainment: Lifelike Autonomus Agents, Communication of the ACM: Special Issue on New Horizons of Commercial and Industrial AI, pp.108-114.

[Ueda, 1996] Ueda, K. and Takagi, Y., 1996, Development of Micro Camera Module, In Proceedings of the 6th Sony Research Forum, pp.114-119.

[Yokote, 1992] Yokote, Y., 1992, The Apertos Reflective Operating System: The Concept and Its Implementation, In Proceeding of the 1992 International Conference of Object-Oriented Programing, System, Languages, and Applications. 